
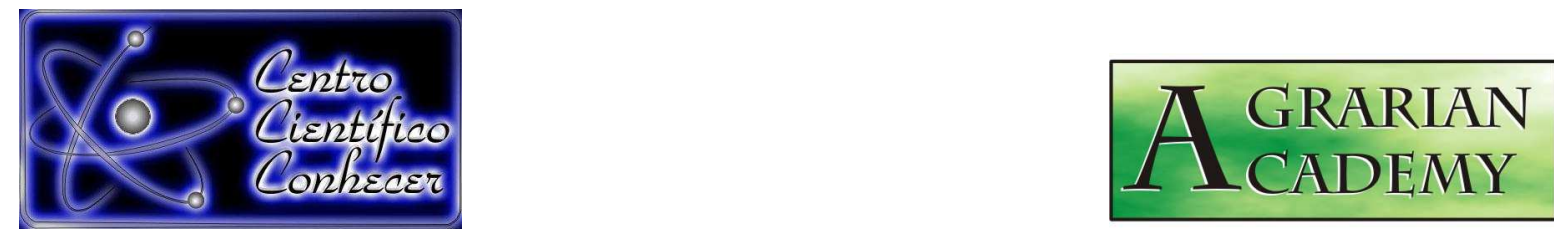

\title{
MODELAGEM GEOESTATíSTICA DA BIOMASSA DO FUSTE DE Araucaria angustifolia EM REMANESCENTE DE FLORESTA OMBRÓFILA MISTA
}

Larissa Ferreira de Lima ${ }^{1}$, Allan Libanio Pelissari², Afonso Figueiredo Filho ${ }^{3}$

1 Graduanda, Faculdade de Engenharia Florestal da Universidade Federal do

Paraná, Curitiba, Paraná, Brasil (larissaferreiralima94@gmail.com)

2 Professor, Departamento de Ciências Florestais da Universidade Federal do

Paraná, Curitiba, Paraná, Brasil

3 Professor, Departamento de Engenharia Florestal da Universidade Estadual do Centro-Oeste, Irati, Brasil

Recebido em: 30/11/2017 - Aprovado em: 15/12/2017 - Publicado em: 31/12/2017 DOI: 10.18677/Agrarian Academy 2017b19

\begin{abstract}
RESUMO
O objetivo deste trabalho foi modelar, mapear e relacionar os padrões e as dinâmicas espaciais do estoque de biomassa do fuste de Araucária angustifolia em um remanescente de Floresta Ombrófila Mista na região Sul do Brasil. A base de dados para a estimativa da biomassa de fuste contou com o diâmetro à altura do peito de trinta indivíduos de Araucaria angustifólia. Realizou-se análise de regressão para dez modelos onde se selecionou o que apresentou menor erro padrão da estimativa, maior coeficiente de determinação ajustado e homogeneidade na distribuição dos resíduos. A biomassa do fuste foi estimada em 400 parcelas de 25 $\mathrm{m} \times 25 \mathrm{~m}$ e submetidas à análise estatística descritiva e ao teste de normalidade de Kolmogorov-Smirnov ao nível de $5 \%$ de significância. As médias apresentaram valores crescentes ao longo do inventário, o que indicou que a floresta não atingiu o seu ponto clímax. As unidades amostrais apresentaram valores semelhantes na distância de alcance, sendo que foram observados valores menores de alcance nos primeiros anos do inventário e maiores nos anos seguintes. O efeito pepita apresentou valores expressivos e o patamar foi alcançado em um mesmo intervalo de valores em todos os anos. Nos mapas temáticos, observou-se que o estoque de biomassa do fuste de Araucaria angustifólia do remanescente aumentou, uma vez que os estoques presentes em classes maiores passaram a apresentar maior quantidade de biomassa. Observou-se também que locais com maior estoque de biomassa do fuste não foram expressivos nos mapas temáticos gerados pela krigagem ordinária pontual.
\end{abstract}

PALAVRAS-CHAVE: distribuição espacial, floresta nacional, mudanças climáticas. 


\title{
GEOESTATISTICAL MODELLING FOR Araucaria angustifolia STEM BIOMASS IN MIXED OMBROPHILOUS FOREST REMNANT
}

\begin{abstract}
The aim of this work was to model, map and correlate the spatial patterns and dynamics of the Araucaria angustifolia stem biomass stock in a Mixed Ombrophilous Forest remnant in Southern Brazil. The database for estimation the stem biomass used the diameter at breast height of thirty Araucaria angustifolia trees. A regression analysis was applied for ten models, where the one with lowest standard error, highest coefficient of adjusted determination and homogeneity of the residues distribution were selected. The stem biomass was estimated in 400 plots of $25 \mathrm{~m} \mathrm{x}$ $25 \mathrm{~m}$ and subjected to descriptive statistical analysis and Kolmogorov-Smirnov normality test at $5 \%$ level of significance. The averages presented increasing values throughout the inventory, which indicated that the forest did not reach its climax point. The plots presented similar values in the distance of reach, being that they were observed smallest values of reach in the first years of the inventory and greater in the following years. The nugget effect presented expressive values and the sill reached same range values in all years. In the thematic maps, it was observed that the Araucaria angustifolia stem biomass stock was increased, since the stocks present in largest classes changed to present a highest biomass quantity. It was also observed that sites with highest biomass stem stock were not expressive in the thematic maps generated by ordinary point kriging.
\end{abstract}

KEYWORDS: spatial distribution, national forest, climate change.

\section{INTRODUÇÃO}

Atualmente, o mundo vive em constante questionamento sobre as atuais mudanças climáticas, cujas causas podem estar relacionadas às atividades antrópicas, como desmatamento, queimadas e emissões de gases do efeito estufa (GEE), ou não antrópicas, sendo causas naturais resultantes das alterações na radiação do sol e dos movimentos orbitais da Terra. Em 1988, a Organização Mundial de Meteorologia (OMM) e o Programa das Nações Unidas para o Meio ambiente (PNUMA) criaram o Intergovernmental Panel on Climate Chance (IPCC). $\mathrm{O}$ IPCC fornece dados científicos sobre as avaliações periódicas das mudanças climáticas, os seus impactos e os riscos futuros, visando formas de adaptação e de mitigação (LEITE, 2015).

As florestas possuem grande importância para mitigação do aquecimento global (MATTSSON et al., 2016; PILLI et al., 2016), pois o combate ao desmatamento contribui para a conservação das florestas naturais e também evita a emissão de gases de efeito estufa para a atmosfera. Com isso, as florestas realizam o estoque de carbono na biomassa, o que consequentemente, evita que $\mathrm{CO}_{2}$ seja emitido, uma vez que é considerado o GEE que mais contribui para o aquecimento global (TOSATO; PELISSARI, 2017).

Para medir e estimar a biomassa presente em um determinado local é necessário determinar o número de árvores a serem amostradas e coletar os dados de diâmetro à altura do peito (DAP) e altura. A estimativa da biomassa pode ocorrer de forma direta e indireta, em que o procedimento indireto está baseado em equações alométricas, sendo essas equações matemáticas que relacionam a 
biomassa com algumas variáveis das árvores, como diâmetro e altura (SILVA; SAMPAIO, 2008).

Ao realizar uma pesquisa referente a trabalhos que estimam a biomassa, notou-se que a maioria dos estudos realizados até o momento estima a biomassa de forma direta. Ratuchne (2010) ajustou e selecionou equações para estimativa da biomassa acima do solo de árvores individuais de uma Floresta Ombrófila Mista Montana no município de General Carneiro por meio do método destrutivo.

Contudo, os trabalhos de estimativa de biomassa não demonstram a dinâmica espacial desse componente nas florestas naturais, apenas quantificam-na como um todo. Assim, a geoestatística é uma ferramenta que permite analisar espacialmente o comportamento da floresta, em que o interpolador de krigagem permite a obtenção de valores em locais não amostrados. Um estudo realizado por Ozturk e Kilic (2016) analisou a diferença na utilização da krigagem sobre interpolação determinística e concluíram a efetividade da geoestatística.

Segundo Isaaks e Srivastava (1989), Webster e Oliver (2007) e Yamamoto e Landim (2013), a fundamentação teórica da geoestatística baseia-se no pressuposto de que unidades amostrais próximas são mais similares entre si em relação àquelas mais distantes no plano espacial. A geoestatística também pode ser aplicada para a verificação do comportamento espacial da estrutura de uma floresta, como em um estudo realizado por Pelissari et al. (2016) em um remanescente natural de floresta Ombrófila Mista.

Com isso, o presente trabalho objetivou modelar, mapear e relacionar os padrões e as dinâmicas espaciais do estoque de biomassa do fuste de Araucária angustifolia (Bertol.) Kuntze em um remanescente de Floresta Ombrófila Mista na região Sul do Brasil. Como hipótese, considerou-se que o uso geoestatística permite obter estimativas da distribuição espacial da biomassa como subsídio à conservação das florestas naturais.

\section{MATERIAL E MÉTODOS}

\section{Local de estudo}

Para o estudo, foram utilizados dados provenientes de um inventário florestal contínuo executado entre 2002 a 2014, em intervalos de três anos, na Unidade de Conservação da Floresta Nacional de Irati, estado do Paraná, sob as coordenadas $25^{\circ} 01^{\prime} \mathrm{S}$ e $25^{\circ} 40^{\prime} \mathrm{S}$ e $51^{\circ} 11^{\prime} \mathrm{W}$ e $51^{\circ} 15^{\prime} \mathrm{W}$. A região apresenta clima Cfb (Köppen), caracterizado pela ausência de estação seca e presença de verão temperado, com precipitação volumétrica superior a $1.300 \mathrm{~mm}$ ao ano e temperatura média anual em torno de $16^{\circ} \mathrm{C}$ a $18^{\circ} \mathrm{C}$ (ALVARES et al., 2013).

As medições trianuais foram executadas em 25 blocos com $100 \mathrm{~m} \times 100 \mathrm{~m}$, correspondentes a dimensão de um hectare cada, e divididas em 16 subunidades cada de $25 \mathrm{~m} \times 25 \mathrm{~m}$, totalizando 400 parcelas georreferenciadas. A alocação das unidades amostrais contínuas visou abranger áreas de um remanescente de Floresta Ombrófila Mista sob um mosaico de paisagens compostas por solos da classe Latossolo Vermelho Distrófico e isentas de influência antrópica, há aproximadamente, sete décadas.

\section{Estimativa da biomassa do fuste}

Para a estimativa da biomassa do fuste, foram ajustados dez modelos a partir dos dados de 30 indivíduos de Araucaria angustifólia. Os modelos utilizados foram 
obtidos por meio da pesquisa desenvolvida por Ratuchne (2010).

$$
\begin{gathered}
w=\beta_{0}+\beta_{1} d+\beta_{2} d^{2} \\
w=\beta_{0}+\beta_{1} d \\
w=\beta_{0}+\beta_{1} d^{2} \\
w=\beta_{0}+\beta_{1} d^{3} \\
\ln (w)=\beta_{0}+\beta_{1} \ln (d) \\
\ln (w)=\beta_{0}+\beta_{1} \ln \left(d^{2}\right) \\
\ln (w)=\beta_{0}+\beta_{1} \ln \left(d^{3}\right) \\
w=\beta_{0}+\beta_{1} d+\beta_{2} d^{2}+\beta_{3} d^{3} \\
w=\beta_{0}+\beta_{1} d+\beta_{2} d^{2}+\beta_{3} d^{3}+\beta_{4} d^{4} \\
w=\beta_{0}+\beta_{1} d+\beta_{2} d^{2}+\beta_{3} d^{3}+\beta_{4} d^{4}+\beta_{5} d^{5}
\end{gathered}
$$

Em que: $w$ = biomassa do fuste $\left(\mathrm{Mg} \mathrm{ha}^{-1}\right) ; d=$ diâmetro a $1,3 \mathrm{~m}$ de altura do solo $(\mathrm{cm}) ; \beta_{i}=$ coeficiente de regressão; e $\ln =$ logaritmo neperiano.

Para a escolha da melhor equação, analisou-se os valores do erro padrão da estimativa em porcentagem (Syx\%), coeficiente de determinação ajustado $\left(R^{2} a j.\right)$ e gráfico dos resíduos, e foram selecionados os modelos que apresentaram os seguintes critérios de seleção: menor valor de Syx\%, maior valor de $\mathrm{R}^{2} a j$. e homogeneidade na distribuição dos resíduos. Utilizou-se o modelo que apresentou os melhores indicadores dos critérios de seleção para estimativa da biomassa do fuste. Estimou-se biomassa do fuste $\left(\mathrm{Mg} \mathrm{ha}^{-1}\right)$ nos anos de 2002 a 2014, com intervalos de três anos, de um inventário contínuo realizado na área de estudo.

\section{Modelagem geoestatística da biomassa do fuste}

As estimativas de biomassa do fuste de Araucaria angustifolia por subunidade amostral foram submetidas à análise estatística descritiva, determinando os valores médios e de coeficiente de variação. Além disso, a normalidade dos dados foi examinada por meio do teste de Kolmogorov-Smirnov ao nível de $5 \%$ de significância.

A análise geoestatística foi utilizada posteriormente para modelar os padrões espaciais da biomassa do fuste, por meio do cálculo das semivariâncias (2), considerando o posicionamento geográfico central de cada subunidade amostral no campo e as distâncias $(h)$ entre elas, bem como as diferenças numéricas $(Z)$ na malha amostral.

$\gamma(h)=\frac{1}{2 N(h)} \sum_{i=1}^{N(h)}\left\{\left[Z\left(x_{i}\right)-Z\left(x_{i}+h\right)\right]^{2}\right\}$

Em que: $\gamma(h)=$ semivariância da variável $Z\left(x_{i}\right) ; h=$ distância; e $N(h)=$ número de pares de pontos medidos $Z\left(x_{i}\right)$ e $Z\left(x_{i}+h\right)$, separados por uma distância $h$.

As semivariâncias foram determinadas entre as subunidades amostrais pontos amostrais em quatro direções no plano: $0^{\circ}(\mathrm{S}-\mathrm{N})$; $45^{\circ}$ (SO-NE); $90^{\circ}(\mathrm{L}-\mathrm{O})$; e $135^{\circ}$ (NO-SE), por meio das quais foram obtidas as semivariâncias médias entre as distâncias equivalentes, além da quantificação dos pares de unidades amostrais computadas. Logo, para as estimativas das semivariâncias em quaisquer distâncias, 
foram ajustados os seguintes modelos de semivariogramas teóricos: esférico (12), exponencial (13) e gaussiano (14), com o auxílio do programa GS+.

$$
\begin{array}{r}
\gamma(h)=\left\{\begin{array}{lr}
C_{0}+C\left[\left(\frac{3}{2}\right)\left(\frac{h}{A c}\right)-\left(\frac{1}{2}\right)\left(\frac{h}{A c}\right)^{3}\right] & \text { se } h \leq A C \\
C_{0}+C & \text { se } h>A C
\end{array}\right. \\
\gamma(h)= \begin{cases}C_{0}+C\left(1-e^{-h / A c}\right) & \text { se } h \leq A C \\
C_{0}+C & \text { se } h>A C\end{cases} \\
\gamma(h)= \begin{cases}C_{0}+C\left(1-e^{-h^{2} / A c^{2}}\right) & \text { se } h \leq A C \\
C_{0}+C & \text { se } h>A C\end{cases}
\end{array}
$$

Em que: $\gamma(h)=$ semivariância da variável $z\left(x_{i}\right) ; h=$ distância; $C_{0}=$ efeito pepita; $C=$ variância a priori dos dados; $C_{0}+C=$ patamar; e $A C=$ alcance.

O semivariograma teórico foi composto pelo efeito pepita $\left(C_{0}\right)$ : semivariância para a distância zero; patamar $\left(C_{0}+C\right)$ : estabilização do semivariograma próxima a variância amostral dos dados; variância a priori $(C)$ : diferença entre $C_{0}+C$ e $C_{0}$; e alcance $(A c)$ : distância onde o semivariograma alcança o patamar, indicando o limite em que as subunidades amostrais são espacialmente correlacionadas (PELISSARI et al., 2014).

A avaliação e a seleção dos melhores ajustes dos semivariogramas experimentais foram baseadas no maior coeficiente de determinação $\left(R^{2}\right)$ e na menor soma de quadrados dos desvios ponderados (SQDP). Enquanto a krigagem ordinária pontual foi aplicada para a geração dos mapas temáticos da biomassa do fuste de Araucária angustifolia, visando avaliar a dinâmica espaço-temporal entre as ocasiões do inventário contínuo.

\section{RESULTADOS E DISCUSSÃO}

Os modelos levantados para a realização da estimativa da biomassa do fuste apresentavam apenas 0 diâmetro à altura do peito (DAP) como variável independente, pois consiste na única medida coletada em campo. Geralmente, o DAP das árvores é usado como a principal variável para a estimativa do estoque em biomassa e carbono de florestas via meios não-destrutivos e equações alométricas (HUY et al., 2016; MATSSON et al., 2016).

Foram encontrados 10 modelos que contavam com a presença de apenas o DAP na equação. Na sequência, estimou-se a biomassa do fuste por meio da análise de regressão de 30 indivíduos de Araucaria angustifólia provenientes da área de estudo. O modelo 4 apresentou os melhores indicadores estatísticos, como menor valor de Syx\%, maior valor de $\mathrm{R}^{2} a j$. e homogeneidade na distribuição dos resíduos para a estimativa da biomassa do fuste das parcelas do inventário contínuo (Tabela 1). 
TABELA 1 Estatísticas dos modelos ajustados para estimativa da biomassa do fuste de Araucaria angustifolia em um remanescente de Floresta Ombrófila Mista.

\begin{tabular}{ccccccccc}
\hline Modelo & $\beta_{0}$ & $\beta_{1}$ & $\beta_{2}$ & $\beta_{3}$ & $\beta_{4}$ & $\beta_{5}$ & $\mathrm{R}^{2}$ aj. & $\mathrm{S}_{\mathrm{yx}} \%$ \\
\hline 1 & 137,016 & $-15,582$ & 0,667 & - & - & - & 0,871 & $20,40 \%$ \\
2 & $-232,410$ & 16,664 & - & - & - & - & 0,846 & $22,30 \%$ \\
3 & $-43,355$ & 0,347 & - & - & - & - & 0,869 & $20,60 \%$ \\
4 & 19,593 & 0,0092 & - & - & - & - & 0,876 & $20,00 \%$ \\
5 & $-2,864$ & 2,477 & - & - & - & - & 0,872 & $20,30 \%$ \\
6 & $-2,864$ & 1,239 & - & - & - & - & 0,872 & $20,30 \%$ \\
7 & $-2,864$ & 0,826 & - & - & - & - & 0,872 & $20,30 \%$ \\
8 & 34643,881 & $-5165,661$ & 256,486 & $-4,220$ & - & - & 0,866 & $20,80 \%$ \\
9 & 1129122,7374 & $-225405,7055$ & 16835,6815 & $-557,5873$ & 6,910 & - & 0,862 & $21,10 \%$ \\
10 & $-11234969,372$ & 2858663,410 & $-290347,112$ & 14714,741 & $-372,111$ & 3,756 & 0,857 & $21,40 \%$ \\
\hline
\end{tabular}

Em que: $\beta_{i}=$ coeficiente de regressão; $\mathrm{R}^{2}$ aj. = coeficiente de determinação ajustado; e $\mathrm{S}_{\mathrm{yx}} \%$ = erro padrão da estimativa em porcentagem.

Analisou-se também a distribuição dos resíduos dos dois melhores modelos, os quais foram designados pela identificação 4 e 6 , respectivamente na Figuras $1 \mathrm{~A}$ e 1B. Após a escolha do modelo, estimou-se a biomassa do fuste de cada indivíduo de Araucaria angustifólia presente nas parcelas de $25 \mathrm{~m} \times 25 \mathrm{~m}$, em que a grande maioria das parcelas contaram com a presença de mais que um indivíduo da espécie e, com isso, realizou-se a soma da biomassa de todos os indivíduos pertencente, e extrapolou-se o estoque de biomassa do fuste para a unidade de um hectare.
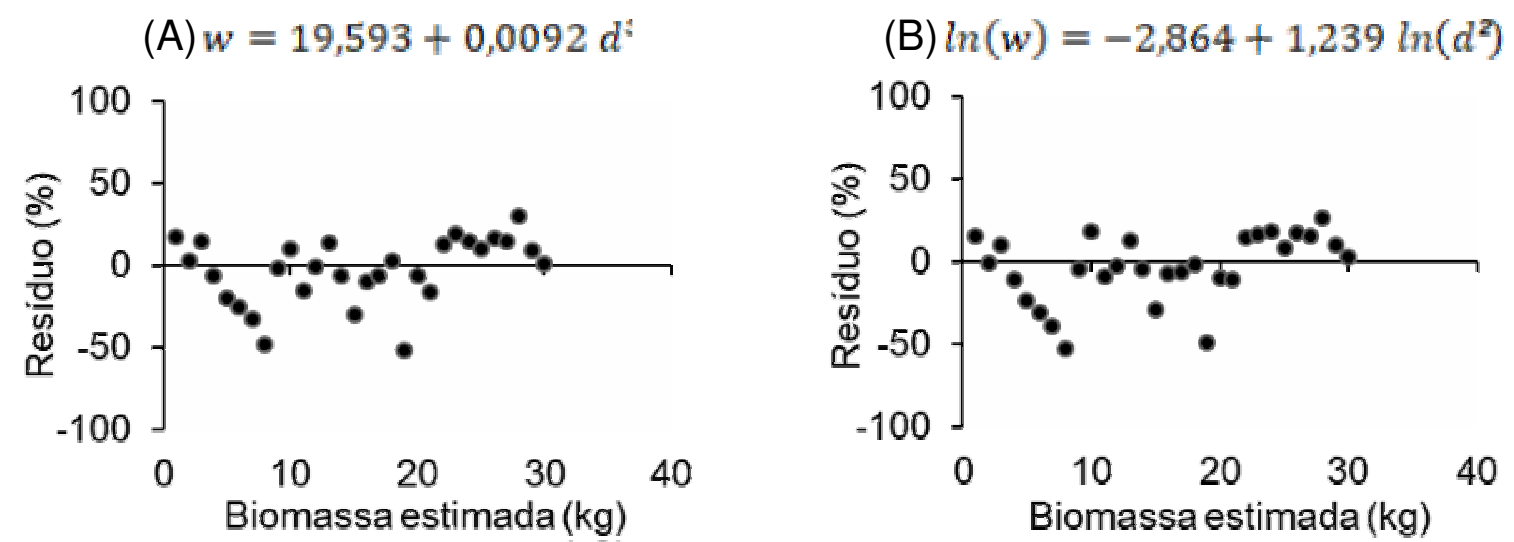

FIGURA 1 Resíduos dos melhores modelos para estimativa da biomassa do fuste de Araucaria angustifolia em um remanescente de Floresta Ombrófila Mista.

Observou-se, por meio das médias (Tabela 2), que o estoque de biomassa do fuste aumentou ao longo do intervalo de 12 anos do inventário contínuo, o que indica que a floresta ainda não atingiu o ponto clímax, o qual consiste na estabilidade dos dados. Em todos os anos, o coeficiente de variação (CV\%) apresentou valores superiores a $100 \%$, demonstrando a elevada variabilidade numérica da variável. O teste K-S indicou não significância ao nível de $5 \%$ de probabilidade, concluindo-se que a biomassa apresenta distribuição normal. 
TABELA 2 Estatísticas descritivas da estimativa da biomassa do fuste de Araucaria angustifolia em um remanescente de Floresta Ombrófila Mista.

\begin{tabular}{cccccc}
\hline Medida & 2002 & 2005 & 2008 & 2011 & 2014 \\
\hline Média (Mg ha-1 $\left.^{-1}\right)$ & 49,74 & 54,73 & 57,11 & 60,01 & 63,20 \\
CV\% & 106,0 & 104,2 & 103,1 & 101,6 & 101,1 \\
K-S & $0,068^{\text {ns }}$ & $0,068^{\text {ns }}$ & $0,068^{\text {ns }}$ & $0,068^{\text {ns }}$ & $0,068^{\text {ns }}$ \\
\hline
\end{tabular}

Em que: $\mathrm{CV} \%=$ coeficiente de variação em porcentagem; $\mathrm{K}-\mathrm{S}=$ teste de Kolmogorov-Smirnov; e ns = não significativo ao nível de $5 \%$ de probabilidade pelo teste K-S (distribuição normal).

As estimativas das semivariâncias em quaisquer distâncias foram ajustadas com o auxílio do programa GS+ para os modelos de semivariogramas teórico, ocorrendo à predominância do modelo esférico, sendo o modelo que apresentou maior coeficiente de determinação $\left(R^{2}\right)$ e menor soma de quadrados dos desvios ponderados (SQDP) após o ajuste (Tabela 3).

TABELA 3 Parâmetros do semivariograma ajustados para estimativa espacial da biomassa do fuste de Araucaria angustifolia em um remanescente de Floresta Ombrófila Mista.

\begin{tabular}{ccccccc}
\hline Ano & Modelo & $\mathrm{C}_{0}$ & $\mathrm{C}_{0}+\mathrm{C}$ & $\mathrm{Ac}$ & $\mathrm{R}^{2}$ & $\mathrm{SQR}$ \\
\hline 2002 & Esférico & 2.000 & 2.540 & 74 & 0,654 & 22.730 \\
2005 & Esférico & 2.400 & 3.000 & 70 & 0,693 & 31.538 \\
2008 & Esférico & 2.800 & 3.150 & 110 & 0,647 & 45.260 \\
2011 & Esférico & 2.950 & 3.400 & 105 & 0,591 & 53.706 \\
2014 & Esférico & 3.200 & 3.730 & 100 & 0,590 & 75.852 \\
\hline
\end{tabular}

Em que: $\mathrm{C}_{0}=$ efeito pepita; $\mathrm{C}_{0}+\mathrm{C}=$ patamar; $\mathrm{Ac}=$ alcance $(\mathrm{m}) ; \mathrm{R}^{2}=$ coeficiente de determinação; e SQR = Soma dos quadrados dos resíduos.

Os coeficientes de determinação $\left(R^{2}\right)$ foram moderados durante todo 0 inventário contínuo, apresentando valores entre zero e um. O alcance (Ac) indicou o limite da correlação espacial entre as parcelas, em que os valores se mostraram menores nos anos iniciais e maiores nos últimos anos da realização do inventário contínuo. Com isso, as parcelas apresentaram distâncias de correlação espacial menores nos primeiros anos e maiores nos posteriores (PELISSARI et al., 2017).

$\mathrm{Na}$ sequência, observou-se o ajuste dos semivariogramas por meio do modelo esférico, o qual foi selecionado como o estatisticamente mais adequado (Figura 2). Esse modelo é comumente o mais utilizado e selecionado nas modelagens geoestatísticas aplicadas em florestas naturais e povoamentos florestais (PELISSARI et al., 2014; PELISSARI et al., 2016; ROVEDA et al., 2016; PELISSARI et al., 2017), o que demonstra a sua elevada flexibilidade para explicar a variabilidade espacial de variáveis dendrométricas. 
(A) 2002

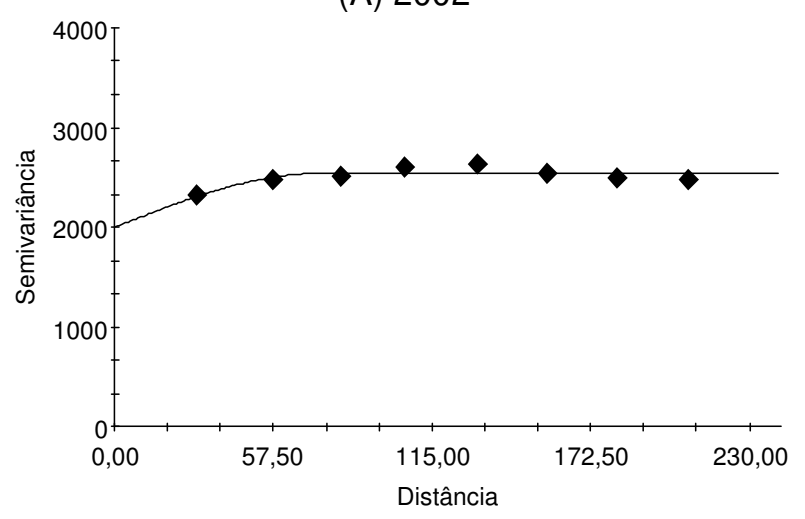

(C) 2008

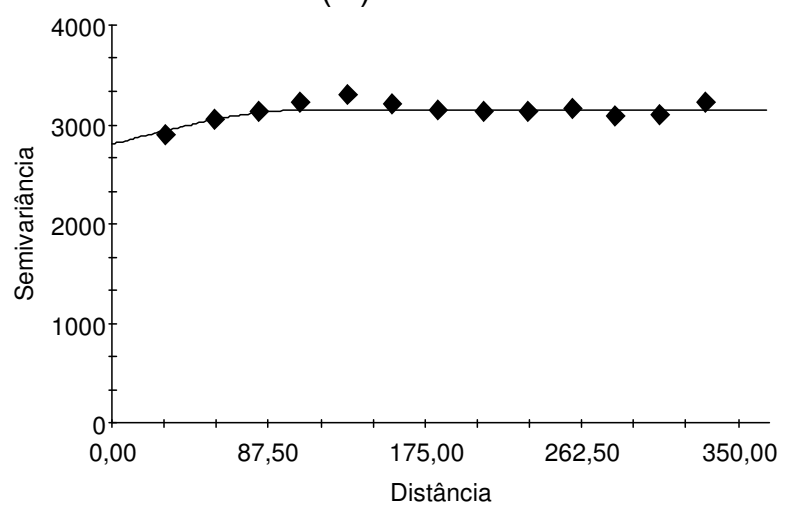

(E) 2014

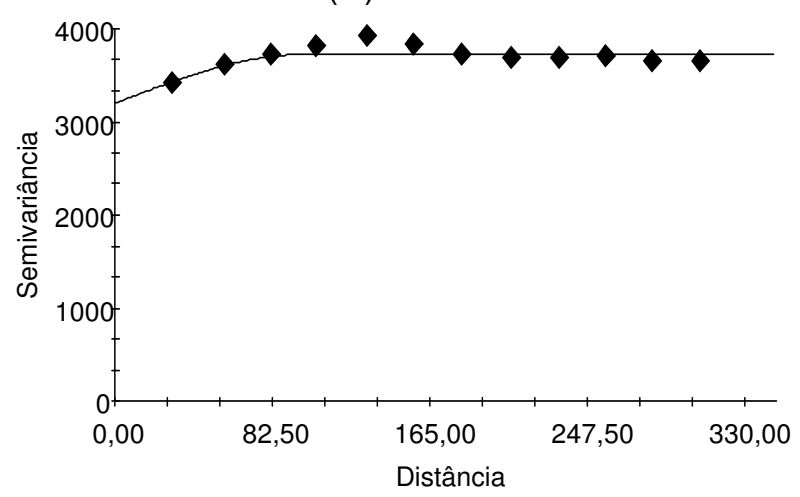

(B) 2005

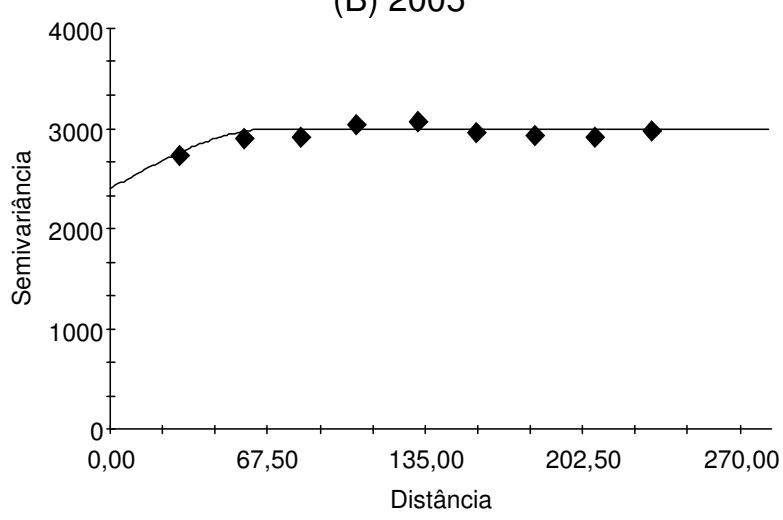

(D) 2011

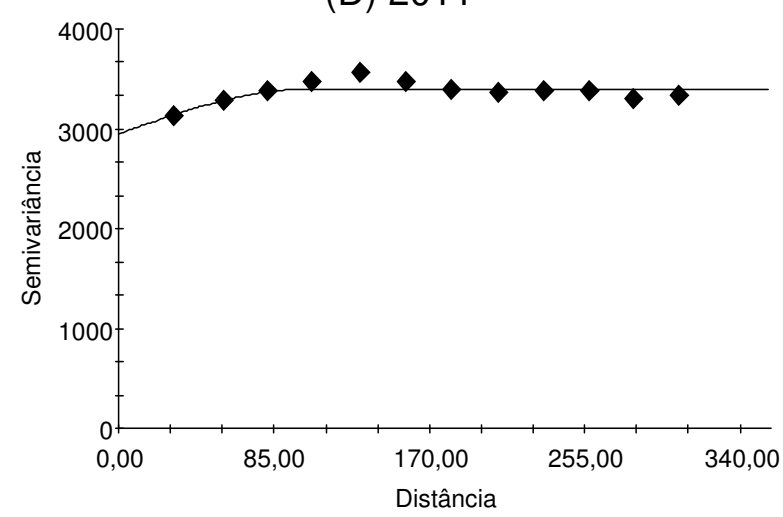

FIGURA 2 Semivariogramas selecionadas para estimativa da biomassa do fuste de Araucaria angustifolia em um remanescente de Floresta Ombrófila Mista.

Os semivariogramas apresentaram alcance (Ac) entre 170 e $180 \mathrm{~m}$, a qual indicou a distância em que as amostras se apresentam correlacionadas espacialmente. $\mathrm{O}$ efeito pepita $\left(\mathrm{C}_{0}\right)$ apresentou valores altos, sendo que $\mathrm{C}_{0}$ próximos a zero são considerados os ideais, os quais podem indicar a influência de efeitos naturais ou artificiais do fenômeno espacial (CARRASCO, 2010), como a presença de maior variabilidade em pequena escala. Além disso, demonstrou crescimento à medida que o estoque de biomassa aumentou, assim como observado por Fox et al. (2007) para diâmetro de árvores. No entanto, a tendência crescente das semivariâncias ao longo da distância, e a subsequente estabilização do alcance, mostra a efetividade da análise aplicada (PELISSARI et al., 2016; ROVEDA et al., 2016).

Os mapas temáticos da biomassa do fuste de Araucaria angustifolia foram AGRARIAN ACADEMY, Centro Científico Conhecer - Goiânia, v.4, n.8; p.190 2017 
gerados por meio da aplicação da krigagem ordinária pontual (Figura 3). Assim, observou-se que, entre as coordenadas X-425 m a X-850 m, o estoque de biomassa do fuste incrementou da classe de $>41 \mathrm{Mg} \mathrm{ha}^{-1}$ para a classe de $>81 \mathrm{Mg} \mathrm{ha}^{-1}$, pois houve 0 aumento de uma mancha aparente. Entre as coordenadas de aproximadamente X-1.000 $\mathrm{m}$ a X-1.275 m, ocorreu diminuição de uma mancha, nessa situação o estoque se mostrou inferior de $41 \mathrm{Mg} \mathrm{ha}^{-1}$. As classes > $122 \mathrm{Mg} \mathrm{ha}^{-}$ 1 não são aparentes nos mapas temáticos, representando pequenos fragmentos compostos por poucos, porém maiores, indivíduos de Araucaria angustifolia.

(A) 2002

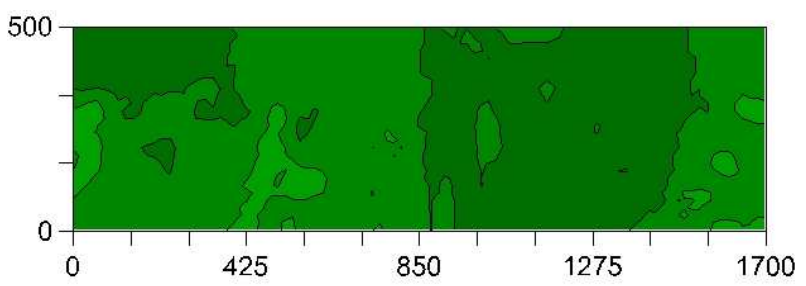

(C) 2008

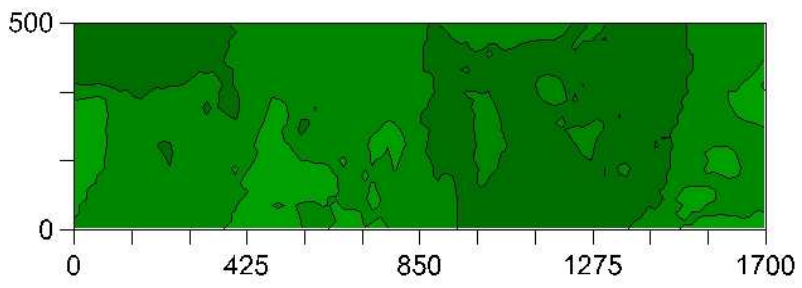

(E) 2014

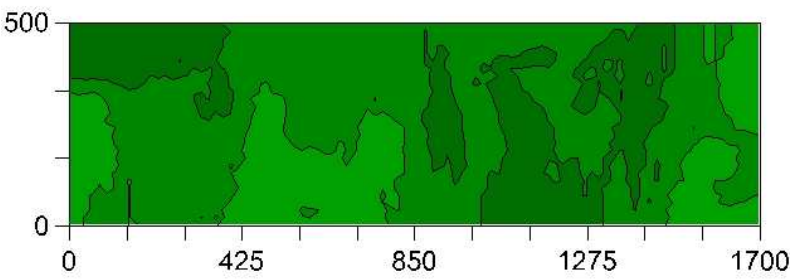

(B) 2005

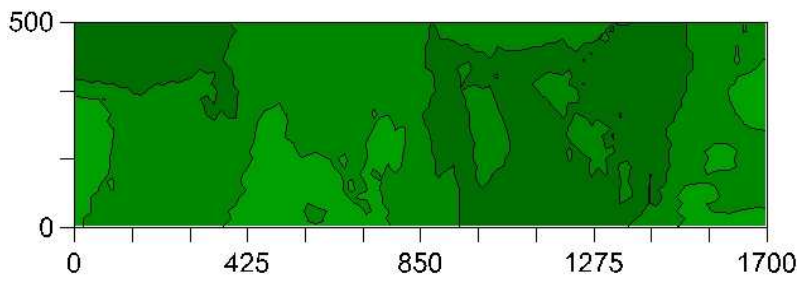

(D) 2011

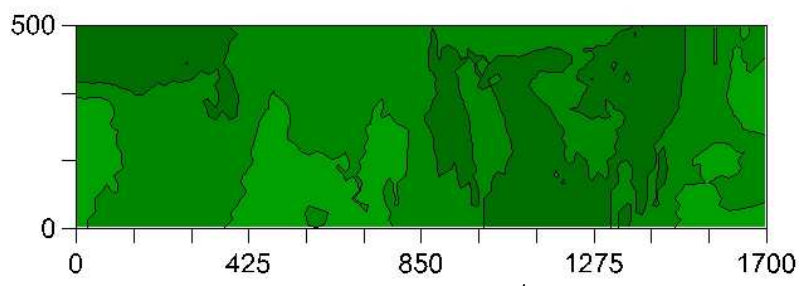

Legenda $\left(\mathrm{Mg} \mathrm{ha}^{-1}\right)$ :
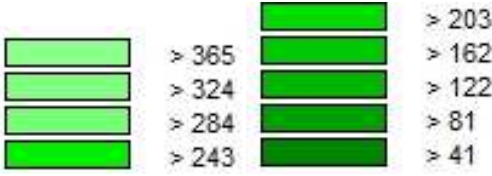

FIGURA 3 Mapas temáticos do estoque de biomassa do fuste de Araucaria angustifolia em um remanescente de Floresta Ombrófila Mista.

A distribuição espacial Araucaria angustifolia no remanescente florestal é formada por um mosaico heterogêneos (PELISSARI et al., 2016), em que as características espaciais estão diretamente relacionadas com as dimensões diamétricas dos indivíduos, bem como as taxas de incremento em biomassa nas diferentes ocasiões do inventário florestal contínuo.

Como a espécie Araucaria angustifolia pode assumir características heliófitas e ombrófilas, o que possibilita o seu enquadramento em grupos pioneiros e climácicos, a sucessão ecológica das folhosas sobre as coníferas acarreta em alterações na estrutura da floresta. Assim, as mudanças nos padrões espaciais do estoque de biomassa da espécie podem indicar avanços sucessional da floresta (PELISSARI et al., 2017).

Nesse sentido, o setor florestal brasileiro possui elevado potencial para a gerar ações mitigadoras do efeito estufa (TOSATO; PELISSARI, 2017). No entanto, novas pesquisas se mostram necessárias para que práticas de manejo e de conservação florestal sejam planejadas em adequação às medidas de combate ao aquecimento global em pequena e larga escalas (PELISSARI et al., 2017). 


\section{CONCLUSÕES}

No presente trabalho é possível constatar a efetividade da geoestatística para modelar, mapear e relacionar os padrões e as dinâmicas espaciais do estoque de biomassa do fuste de Araucária angustifolia em um remanescente de Floresta Ombrófila Mista na região Sul do Brasil. A biomassa do fuste apresenta padrões espaciais que expressam o aumento do estoque ao longo do inventário contínuo.

\section{REFERÊNCIAS}

ALVARES, C. A.; STAPE, J. L.; SENTELHAS, P. C.; GONÇALVES, J. L. de M.; SPAROVEK, G. Köppen's climate classification map for Brazil. Meteorologische Zeitschrift, $\quad$ v. 22, p. 1-18, 2013. Disponível em: <https://www.schweizerbart.de/papers/metz/detail/22/82078/Koppens_climate_classif ication_map_for_Brazil>.doi: 10.1127/0941-2948/2013/0507

CARRASCO, P. C. Nugget effect, artificial or natural? The Journal of The Southern African Institute of Mining and Metallurgy, v. 110, p. 299-305, 2010.

FOX, J. C.; BIC, H.; ADES, P. K. Spatial dependence and individual-tree growth models. Forest Ecology and Management, v. 245, p. 10-19, 2007.

HUY, B.; KRALICEK, K.; POUDEL, K. P.; PHUONG, V. T.; KHOA, P. V.; HUNG, N. D.; TEMESGEN, H. Allometric equations for estimating tree aboveground biomass in evergreen broadleaf forests of Viet Nam. Forest Ecology and Management, v. 382, p. 193-205, 2016. Disponível em: <http://dx.doi.org/10.1016/j.foreco.2016.10.021>. doi: 10.1016/j.foreco.2016.10.021

ISAAKS, E. H.; SRIVASTAVA, R. M. Applied geostatistics. Oxford University Press, 1989. $561 \mathrm{p}$.

LEITE, J. C. Controvérsias na climatologia: o IPCC e o aquecimento global antropogênico. Scientiae Studia, v. 13, n. 3, p. 643-77, 2015. Disponível em: <http://dx.doi.org/10.1590/S1678-31662015000300008>. doi: 10.1590/S167831662015000300008

MATTSSON, E.; OSTWALD, M.; WALLIN, G.; NISSANKA, S. P. Heterogeneity and assessment uncertainties in forest characteristics and biomass carbon stocks: Important considerations for climate mitigation policies. Land Use Policy, v. 59, p. 84-94, 2016. Disponível em: <http://dx.doi.org/10.1016/j.landusepol.2016.08.026>. doi: 10.1016/j.landusepol.2016.08.026

OZTURK, D.; KILIC, F. Geostatistical Approach for Spatial Interpolation of Meteorological Data. Anais da Academia Brasileira de Ciências, v. 8, n. 4, p. 2121-2136, 2016. Disponível em: <http://dx.doi.org/10.1590/00013765201620150103>. doi: 10.1590/0001-3765201620150103

PELISSARI, A. L.; FIGUEIREDO FILHO, A.; CALDEIRA, S. F.; MACHADO, S. A. Geostatistic applied to spatial modeling of hypsometric relationships in forest stands. American International Journal of Contemporary Research, v. 4, p. 67-76, 2014. Disponível em: < http://www.aijcrnet.com/journals/Vol_4_No_7_July_2014/9.pdf>. 
PELISSARI, A. L.; FIGUEIREDO FILHO, A.; SANQUETTA, C. R.; EBLING, A. A.; ROVEDA M.; CYSNEIROS, V. C. Estrutura espacial arbórea de um remanescente natural de floresta ombrófila mista. BIOFIX Scientific Journal, v. 1, n. 1, p. 27-32, 2016. Disponível em: <http://revistas.ufpr.br/biofix/article/view/49094>. doi: 10.5380/biofix.v1i1.49094

PELISSARI, A. L.; FIGUEIREDO FILHO, A.; PÉLLICO NETTO, S.; EBLING, A. A.; ROVEDA, M.; SANQUETTA, C. R. Geostatistical modeling applied to spatiotemporal dynamics of successional tree species groups in a natural Mixed Tropical Forest. Ecological Indicators, v. 78, p. 1-7, 2017. Disponível em: <https://doi.org/10.1016/j.ecolind.2017.02.044>. doi: 10.1016/j.ecolind.2017.02.044

PILLI, R.; GRASSI, G.; KURZ, W. A.; MORIS, J. V.; VIÑAS, R. A. Modelling forest carbon stock changes as affected by harvest and natural disturbances. II. EU-level analysis. Carbon Balance Manage, v. 11, n. 20, p. 1-19, 2016. Disponível em: <https://doi.org/10.1186/s13021-016-0059-4>. doi: 10.1186/s13021-016-0059-4.

RATUCHNE, L. C. Equações alométricas para a estimativa de biomassa, carbono e nutrientes em uma floresta ómbrofila mista. Irati: UNICENTRO, 2010. $111 \mathrm{p}$.

SILVA, G. C.; SAMPAIO, E. V. S. B. Biomassas de partes aéreas em plantas de caatinga. Revista Árvore, v. 32, n. 3, p. 567-575, 2008. Disponível em: <http://dx.doi.org/10.1590/S0100-67622008000300017>. doi: 10.1590/S010067622008000300017

TOSATO, J. P.; PELISSARI, A. L. Inventário de emissões de gás de efeito estufa em empresa de base florestal. BIOFIX Scientific Journal, v. 2, n. 2, p. 53-59, 2017. Disponível em: <http://dx.doi.org/10.5380/biofix.v2i2.55069>. doi: 10.5380/biofix.v2i2.55069

WEBSTER, R.; OLIVER, M. A. Geostatistics for environmental scientists. 2. ed. West Sussex: John Wiley \& Sons Ltd, 2007. 333 p.

YAMAMOTO, J. K.; LANDIM, P. M. B. Geoestatística: conceitos e aplicações. São Paulo: Oficina de textos, 2013. $215 \mathrm{p}$. 